\section{Esterification in ionic liquids: the influence of solvent basicity}

\author{
Thomas P. Wells, Jason P. Hallett, Charlotte K. Williams, *
} Tom Welton*

Department of Chemistry, Imperial College London, South Kensington Campus, London, SW7 2AZ, United Kingdom

t.welton@imperial.ac.uk

RECEIVED DATE

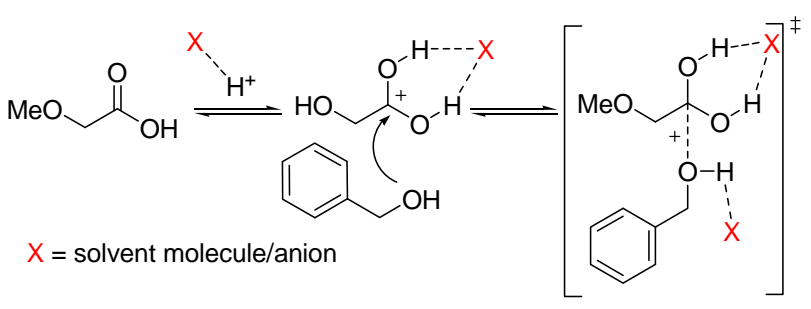

The second order rate constant $\left(\mathrm{k}_{2}\right)$ for the esterification of methoxyacetic acid with benzyl alcohol is reported in a range of ionic and molecular solvents. The solvent effects on esterification rate are examined using a linear solvation energy relationship based on the Kamlet-Taft solvent scales $\left(\alpha, \beta\right.$ and $\left.\pi^{*}\right)$. It is shown that the hydrogen bond basicity of the solvent is the dominant parameter in determining the esterification rate and that the best rates are achieved in low basicity solvents.

Often, the largest contributor to the environmental footprint of a chemical process is the solvent used. Solvents have a significant impact because of the quantity they are used in; for example in pharmaceutical production they typically account for between 80 and $90 \%$ of the mass utilization of a batch operation. ${ }^{1}$ Consequently, replacing conventional solvents, usually volatile organic compounds (VOCs), with more environmentally benign media, or with no solvent at all, is one of the central tenets of Green Chemistry ${ }^{2}$ and a subject of significant academic and commercial interest.

In the search for alternative solvents, ionic liquids (ILs) have emerged amongst the front runners due to their unusual and interesting properties. ${ }^{5}$ Firstly, they exhibit negligible vapor pressure at standard conditions, negating concern over atmospheric emissions. They also dissolve a wide range of organic and inorganic materials, have high thermal stability, are liquid over a wide range of temperatures and are generally non-flammable. Thus, despite concerns over their unknown toxicities ${ }^{6}$, they are widely proposed as "green solvents".

Esterification is a fundamental reaction of both academic and industrial chemistry; the production of polyesters alone exceeds 9 million tonnes per year and accounts for 55\% of synthetic fibres produced. ${ }^{7}$ Consequently the novel synthesis of polyesters is a subject of much interest. ${ }^{8}$ In terms of atom economy, the stoichiometric condensation of an acid and alcohol, without prior functionalisation, constitutes an efficient process, with water being the only by-product. However, due to the unfavorable position of the esterification equilibrium, reactive acid derivatives, such as acyl chlorides or anhydrides, are commonly employed and a large excess of one reagent is used to drive the reaction, thus creating waste.

Ionic liquids are useful media for esterification reactions because they enable vacuum (to remove condensate) and solvent to be used together, creating an opportunity to drive the equilibrium. Also, ILs properties can be tuned to be both polar and hydrophobic. ${ }^{9}$ This points to the intriguing possibility of being able to dissolve polar solutes in a dehydrating solvent. For example, ILs have been shown to dissolve a wide range of carbohydrates, a class of molecules gaining importance as a chemical feedstock. ${ }^{10-13}$ Finally, ILs are commonly referred to as designer solvents. This is because anions and cations can be changed independently of one another, allowing solvent-solute interactions to be tuned to purpose.

Recent work by Tanabe et al. ${ }^{14}$ and Sakakura et al. ${ }^{15}$ has demonstrated the use of biarylammonium salts as mild catalysts for the esterification of acids with alcohols. High conversions were achieved without the removal of water, demonstrating the potential of salts, in conjunction with hydrophobic solvents, to catalyze esterification and drive conversion.

Esterification has already been demonstrated in various ionic liquids. Davis et al., were the first to report the use of IL tethered sulfonic acids to promote Fisher esterification. ${ }^{16,17}$ This concept of a Bronsted IL being used as an esterification catalyst has subsequently been expanded upon, most recently by Li et $a l^{18}$ and Gameshpure et al. ${ }^{19}$ Jiang et al, investigated $p$ TSA catalyzed esterification in imidazolium based $\left[\mathrm{BF}_{4}\right]^{-}$and $\left[\mathrm{PF}_{6}\right]^{-} \mathrm{ILs}$; they observed that the choice of IL had an effect on the equilibrium conversion. ${ }^{20}$ The use of enzymes in ILs for (poly)ester synthesis has also been explored. ${ }^{21,22}$ To date, it has remained unclear which properties of ILs, as solvents, are conducive to rapid esterification kinetics.

We have previously reported kinetic studies of nucleophilic substitution reactions in ionic liquids, rationalizing solvent effects with a Kamlet-Taft linear solvation energy relationship (LSER). ${ }^{23,24}$ Recently, LSER methods have also been applied to ILs in the investigation of chromatography stationary phases, ${ }^{25}$ molar volume ${ }^{26}$ and gas solubilities. ${ }^{27}$ The general form of an LSER is shown in eq 1, where XYZ represents some solvent dependant property (herein, the natural logarithm of the rate constant). The Kamlet Taft approach utilizes three solvent descriptors: $\alpha$ (hydrogen bond acidity), $\beta$ (hydrogen bond basicity), and $\pi^{*}$ (dipolarity/polarizability) as measured by a series of solvatochromic dyes. The coefficients $\mathrm{a}, \mathrm{b}$ and $\mathrm{s}$ are solute specific and measure the sensitivity of a property (XYZ) to $\alpha, \beta$ and $\pi^{*}$. Multi-variant regression can then be used to fit the three solvent parameters $\left(\alpha, \beta\right.$ and $\left.\pi^{*}\right)$ with the experimentally determined rate constants $\left(\ln k_{2}\right)$ by varying the sign and magnitude of the coefficients ( $a, b$ and $s)$.

$$
X Y Z=\text { const }+\alpha \alpha+b \beta+s \pi^{+4}
$$

Here, we report a related approach to rationalize esterification rates in a range of ionic and molecular solvents. It is proposed that this will help us to understand which ionic liquids are most suitable as esterification solvents. 


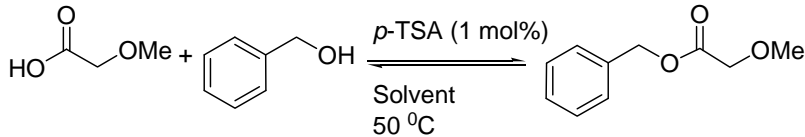

SCHEME 1. Esterification of benzyl alcohol with methoxyacetic acid, at equimolar concentration

The $p$-TSA ( $1 \mathrm{~mol} \%)$ catalyzed esterification between methoxy acetic acid and benzyl alcohol, at $50{ }^{\circ} \mathrm{C}$ (Scheme 1), was studied as a model esterification reaction. $1 \mathrm{M}$ concentrations were used so as to be representative of real synthetic conditions, as opposed to a classical infinite dilution kinetic experiment. The reagents were used in approximately, but not precisely, equimolar amounts; the ratio of initial concentrations and conversions were determined from ${ }^{1} \mathrm{H}$ NMR integrals as discussed in the Supporting Information. The $p$-TSA was selected as the Brønsted acid catalyst due to its good solubility in a wide range of solvents. The reagents were chosen because of their suitability for ${ }^{1} \mathrm{H}$ NMR spectroscopic monitoring; ${ }^{1} \mathrm{H}$ NMR resonances of the reagents are clearly distinguishable from those of the resulting ester and also from those of most common molecular and ionic solvents. This allowed for direct measurement of the reaction conversion without the need for solvent removal.

In situ ${ }^{1} \mathrm{H}$ NMR monitoring was not undertaken as ionic liquids are viscous; therefore the reaction was carried out in a stirred vessel so that diffusion limited kinetics were avoided and aliquots were periodically removed for analysis. At $50{ }^{\circ} \mathrm{C}$, the reaction proceeded at an observable rate in all solvents.

The kinetic measurements were only taken until product concentration was high enough to make the back reaction significant. The point at which this occurred was dependant on the solvent's water miscibility and ranged between 15 and $60 \%$. Simultaneously observing the conversion, $x$, and the initial acid:alcohol ratio, $\theta_{\text {acid, }}$ as experimental ratios of ${ }^{1} \mathrm{H}$ NMR integrals enabled determination of the second order rate constant, $\mathrm{k}_{2}$, from a single reaction (eq 2).

$$
\begin{aligned}
& k_{2} t=\frac{1}{[\text { aloohot] }} \frac{1}{\left(\theta_{\text {avid }}-1\right)} \ln \frac{\left(\theta_{\text {avd }}-x\right)}{\theta_{\text {and }}(1-x)}
\end{aligned}
$$

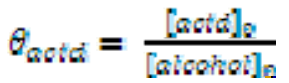

$$
\begin{aligned}
& x=\frac{\text { [esterl] }}{\text { [alohol] }}
\end{aligned}
$$

The rates of esterification were determined for a range of solvents, the corresponding rate constants, their standard deviations $(\sigma)$ and experimentally determined Kamlet-Taft parameters are shown in Table 1 . The reaction was also attempted in DMSO but no reaction was observed in a reasonable timescale and DMSO was excluded from the study. Multivariate regression was used to fit the observed $\ln k_{2}$ values to the Kamlet-Taft LSER. Any coefficients ( $a, b$ or s) with a p-value of over 0.01 were rejected as statistically insignificant and assumed to be zero. The analysis was then repeated without the corresponding parameter $\left(\alpha, \beta\right.$ or $\pi^{*}$ respectively). This process yielded eq 5, which is shown graphically in Figure 1.

The high degree of confidence in the value of $b(-7.25)$ is demonstrated by a p-value of $1.1 \times 10^{-4}$.

TABLE 1: The rate constants $\left(k_{2}\right)$ of esterification and Kamlet-Taft parameters of different solvents

\begin{tabular}{cccccc}
\hline Solvent & $\begin{array}{c}\boldsymbol{k}_{2}\left(\mathbf{M}^{-1} \mathbf{m i n}^{-1}\right) \\
\times \mathbf{1 0}^{\mathbf{4}}\end{array}$ & $\begin{array}{c}\boldsymbol{\sigma}^{\mathrm{a}} \\
\times \mathbf{1 0}^{4}\end{array}$ & $\boldsymbol{\alpha}$ & $\boldsymbol{\beta}$ & $\boldsymbol{\pi}^{*}$ \\
\hline Toluene & 110 & 9.4 & 0.00 & 0.09 & 0.46 \\
Acetonitrile & 16.6 & 0.36 & 0.36 & 0.39 & 0.82 \\
THF & 2.87 & 0.38 & 0.00 & 0.58 & 0.62 \\
\hline & & & & & \\
\hline$\left[\mathrm{C}_{4} \mathrm{C}_{1}\right.$ im $]\left[\mathrm{N}(\mathrm{Tf})_{2}\right]$ & 52.9 & 4.0 & 0.60 & 0.21 & 1.01 \\
{$\left[\mathrm{C}_{4} \mathrm{C}_{1}\right.$ im $][\mathrm{OTf}]$} & 8.60 & 0.71 & 0.60 & 0.51 & 1.03 \\
{$\left[\mathrm{C}_{4} \mathrm{C}_{1} \mathrm{C}_{1}\right.$ im $]\left[\mathrm{N}(\mathrm{Tf})_{2}\right]$} & 44.7 & 1.6 & 0.38 & 0.28 & 1.03 \\
{$\left[\mathrm{C}_{4} \mathrm{C}_{1}\right.$ py $]\left[\mathrm{N}(\mathrm{Tf})_{2}\right]$} & 55.0 & 5.4 & 0.41 & 0.26 & 0.97 \\
\hline
\end{tabular}

a) Rates determined in triplicate

$$
m k_{2}=7.25 \beta-3.59
$$

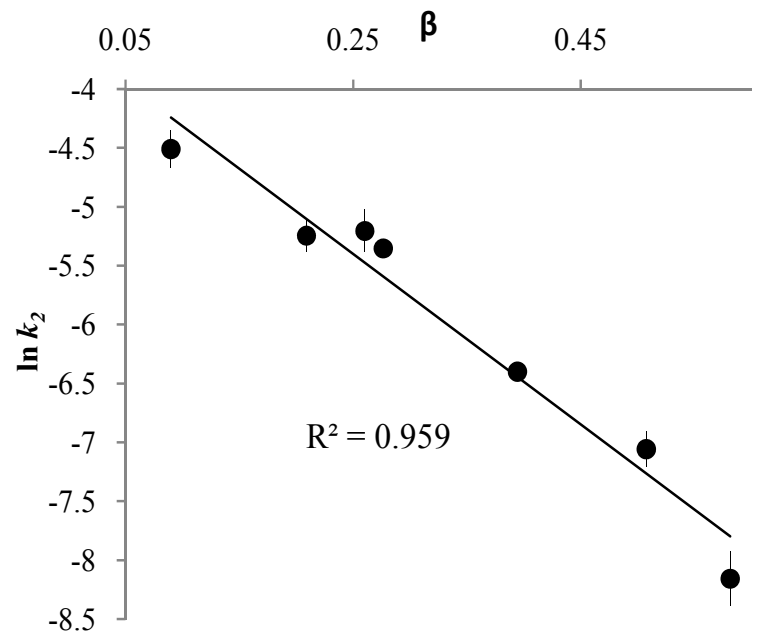

FIGURE 1: Linear plot of $\ln k_{2}$ against $\beta$ values. Error bars denote $2 \sigma, 95 \%$ confidence limit

This analysis indicates that the $\beta$ value of a solvent is the dominant factor in determining the esterification rate. Therefore, the hydrogen bond basicity of a solvent determines the esterification rate and a solvent with low basicity exhibits a high esterification rate. The predictive strength of this theory is portrayed in Figure 2, which shows an excellent correlation between the predicted and experimental values of $\ln k_{2}$. 


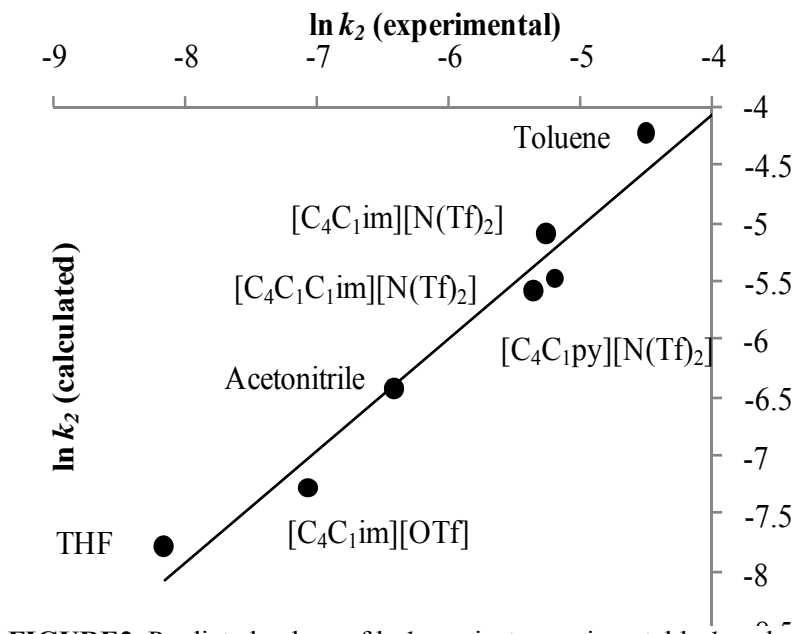

FIGURE2. Predicted values of $\ln k_{2}$ against experimental $\ln k_{2}$ vâlué

Given the observed second order reaction kinetics and the likelihood that the mechanism is the same for ionic and molecular solvents, the most probable mechanism is bimolecular acid-catalyzed acyl-oxygen cleavage $\left(\mathrm{A}_{\mathrm{AC}} 2\right){ }^{28} \mathrm{The}$ rate limiting step (RLS) for the proposed mechanism is shown in Scheme 2. It should be noted that the formation of the activated complex 2 involves the dispersion of charge. The Hughes-Ingold approach to nucleophilic substitutions related solvent polarity to the reaction rate and predicts that a polar solvent would retard esterification rate. Kamlet and Taft separated solvent polarity into three separate components $(\alpha, \beta$ and $\left.\pi^{*}\right)$. Therefore, by analogy with the Hughes-Ingold approach, it is proposed that a hydrogen bond accepting solvent could preferentially stabilize the reacting species $\mathbf{1}$. This stabilization would increase $\Delta \mathrm{G}^{\ddagger}$ and decrease the reaction rate. However, such reasoning fails to explain why the hydrogen bond basicity is so significant while the solvent's acidity and polarizability appear irrelevant. Consequently, this is not thought to be the major effect observed.

SCHEME 2. Rate limiting step for $\mathrm{A}_{\mathrm{AC}} 2$ esterification<smiles>COC(O)(O)CCO</smiles>

1

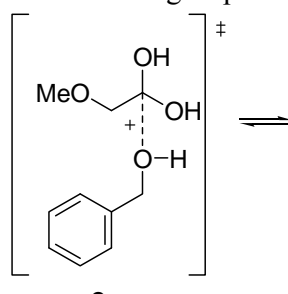

2

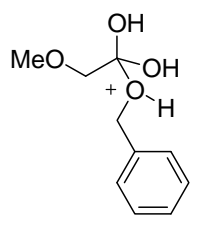

3
Given the relatively high concentrations of acid and alcohol used, self association of the species, as a mechanism of accelerating or decelerating the reaction, cannot be ruled out. To test this, the $k_{2}$ of esterification was determined in toluene at $10 \times$ dilution $(0.1 \mathrm{M})$. Toluene was chosen as it has the lowest values of $\alpha, \beta$ and $\pi^{*}$ and as such should interact least strongly with substrates, therefore promoting self association most strongly. At $0.1 \mathrm{M}$ concentration of each reagent in toluene, a rate constant of $81.3 \times 10^{-4} \mathrm{M}^{-1} \mathrm{~min}^{-1}$ was observed, or $73 \%$ of the original $1.0 \mathrm{M}$ rate. It appears that self association does provide a modest acceleration of rate. Whilst a more basic solvent is likely to reduce the degree of association and thus decelerate the reaction, given the small change in rate upon a $10 \times$ dilution, it is not considered to be a strong enough effect to account for the wide range of rates observed between solvents $(40 \times)$.

The species $\left[\mathrm{HCl}_{2}\right]^{-},\left[\mathrm{H}_{2} \mathrm{Cl}_{3}\right]^{-}$and $\left[\mathrm{H}_{3} \mathrm{Cl}_{4}\right]^{-}$are known to form when Brønsted acids are dissolved in chloride rich chloroaluminate ionic liquids. ${ }^{29,30}$ This suggests that when $p$ TSA dissolves in an ionic solution, the protons will be associated either with the conjugate base, the solvent anions or both. The relative prevalence of each association will depend on the concentration and proton affinity of the species in question. In the same manner, a "free" proton in molecular solvents will be associated, to some degree, with its conjugate base and/or the solvent. The concentration of the conjugate base is constant across the series of solvents under investigation, so the proton affinity of the solvent must be responsible for any differences in the availability of the solvated proton to participate in the RLS of esterification.

Reducing the availability of protons in solution will reduce the equilibrium concentration of the reacting species (1) and lower the rate. The proton affinity of a molecule can be described by its $\mathrm{pK}_{\mathrm{a}}$ or the Gibbs free energy change for its gas phase deprotonation $\left(\Delta \mathrm{G}_{\mathrm{H}}\right)$. It has been previously shown that, for ionic liquids, the $\Delta \mathrm{G}_{\mathrm{H}}$ of an anion is can be correlated with the $\beta$ value of the corresponding ionic liquid. ${ }^{31}$ It follows that a high $\beta$ value corresponds to a high proton affinity and thereby a low proton availability and low rate, as observed. This is evidence for a degree of solvent leveling of protic acids.

Furthermore, Jiang et al., have demonstrated that the equilibrium esterification conversion was significantly higher in hydrophobic $\left[\mathrm{PF}_{6}\right]^{-}$based ILs than in hydrophilic $\left[\mathrm{BF}_{4}\right]^{-}$ based ILs. ${ }^{20}$ This is consistent with the hydrogen bond basicity $(\beta)$ of an ionic liquid, controlled by the anion, determining water miscibility, with a high $\beta$ value corresponding to high water miscibility. ${ }^{9}$ It follows that a low $\beta$ solvent will not only yield a higher reaction rate, but a greater equilibrium conversion too.

In summary, the second order rate constant $\left(\mathrm{k}_{2}\right)$ for the esterification of methoxy acetic acid with benzyl alcohol in a range of solvents has been determined directly using ${ }^{1} \mathrm{H}$ NMR spectroscopy and the integrated second order rate law (Eq 2). It has been shown that the $\beta$ value of the solvent is the dominant parameter in the LSER for the $\ln k_{2}$ of esterification. Therefore, it is concluded that solvent basicity determines esterification rate and that the best rates are achieved in low basicity solvents. This can be rationalized in terms of the solvent's proton affinity determining the availability of the catalytic proton to participate in the RLS of $\mathrm{A}_{\mathrm{AC}} 2$, as the major factor behind the observed solvent dependency. Thus, a low basicity solvent, as show by its $\beta$ value, should be chosen to optimize the esterification rate, provided that the reagent solubility can be maintained.

\section{Experimental Section}

Materials and Reagents All reagents were purchased from commercial suppliers. Unless otherwise stated, solvents and reagents were freshly distilled and dried using standard procedures.

Synthesis of Ionic Liquids As previously reported, ${ }^{9}$ except that ethyl acetate was used in place of toluene in an improved synthesis of $\left[\mathrm{C}_{4} \mathrm{C}_{1} \mathrm{im}\right] \mathrm{Cl}$ (see supporting information).

Kamlet Taft Measurements As previously reported. ${ }^{31}$

Esterification kinetics The procedure was carried out using a Carousel 12 Tube stirrer hot plate, with a reflux condenser and under a nitrogen atmosphere. Three reaction tubes were 
purged for 30 mins with $\mathrm{N}_{2}$. Under a dry atmosphere, $p$-TSA $(0.076 \mathrm{~g}, 0.400 \mathrm{mmol})$ was added to a volumetric flask and made up to $10 \mathrm{~mL}$ with diethyl ether. An accurate aliquot of this solution $(250 \mu \mathrm{L})$ was added by volumetric syringe to each tube. The tubes were heated at $50{ }^{\circ} \mathrm{C}$ and placed under an aspirator vacuum for 2 hours to remove the diethyl ether. A volumetric flask was purged with $\mathrm{N}_{2}$ for $30 \mathrm{mins}$ and methoxyacetic acid $(0.360 \mathrm{~g}, 4 \mathrm{mmol})$ was added and made up to $2 \mathrm{~mL}$ with the solvent under investigation. Accurate aliquots of this solution (490, 500 and $510 \mu \mathrm{L}$ respectively) were added to each tube by volumetric syringe and allowed time to warm to $50^{\circ} \mathrm{C}$. A volumetric flask was purged with $\mathrm{N}_{2}$ for $30 \mathrm{mins}$ and benzyl alcohol $(0.430 \mathrm{~g}, 4 \mathrm{mmol})$ was added and made up to $2 \mathrm{~mL}$ with the solvent under investigation. Accurate aliquots of this solution $(510,500$ and $490 \mu \mathrm{L}$ respectively) were added to each tube by volumetric syringe. The point of injection was deemed to be $t=0$. Regular samples were taken from each tube and analyzed by ${ }^{1} \mathrm{H}$ NMR spectroscopy until the reaction reached equilibrium.

Preparation of NMR samples Small volumes of the reaction mixture (ca $0.05 \mathrm{~mL})$ were dissolved in $\mathrm{CDCl}_{3}(0.5$ $\mathrm{mL}) .{ }^{1} \mathrm{H}$ NMR spectra were recorded on a $400 \mathrm{MHz}$ spectrometer. A $10 \mathrm{~s}$ delay time was applied to ensure complete relaxation and that accurate integral values were recorded.

Acknowledgements. We would like to thank the EPSRC and the Department of Chemistry at Imperial College London for a DTA studentship (TPW) and the Marshall-Sherfield Foundation for a research fellowship (JPH). We would also like to thank Stephen Boyer, London Metropolitan University, for Elemental Analysis, John Barton, Imperial College London, for Mass Spectrometry and Pete Haycock and Dick Shepard, Imperial College London, for NMR support.

Supporting Information Available: Synthesis and spectroscopic characterization of ILs, kinetic data, integrated rate law derivation, method of calculating $\mathrm{x}, \theta_{\text {acid }}$ and $\mathrm{k}_{2}$. This material is available free of charge via the Internet at http://pubs.acs.org.

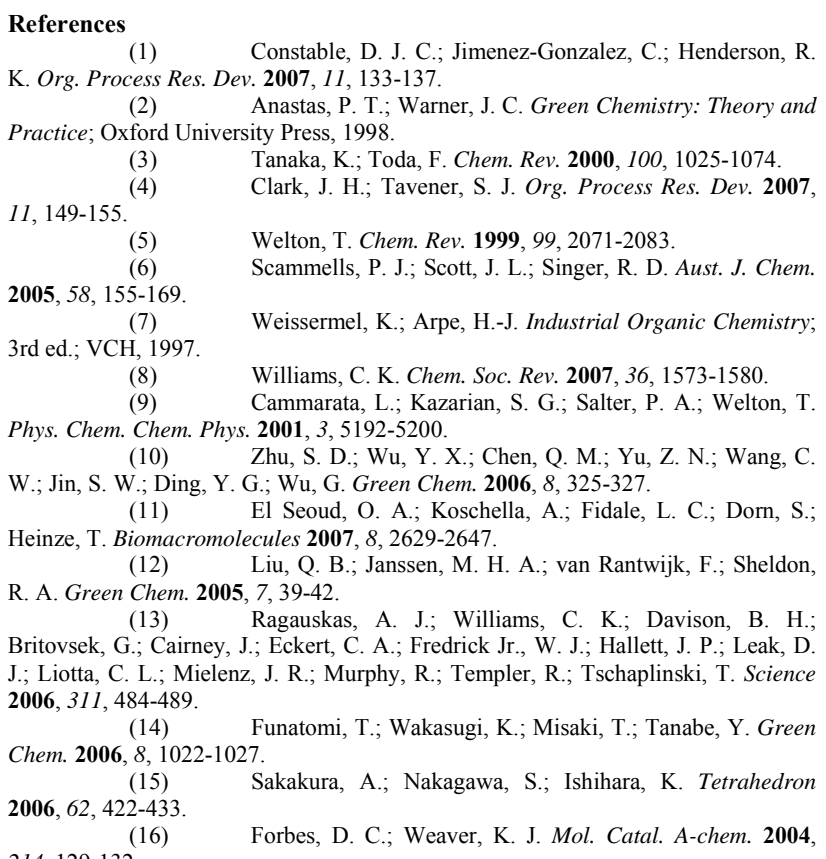

(17) Cole, A. C.; Jensen, J. L.; Ntai, I.; Tran, K. L. T.; Weaver, C.; Toc. 2002, 124, 5962-5963.

$\begin{array}{lll}\text { 164. } & \text { Li, X. Z.; Eli, W. J. Mol. Catal. A-chem. 2008, 279, 159 } \\ & (19) & \text { Ganeshpure, P. A.; George, G.; Das, J. Mol. Catal. A-chem }\end{array}$

2008, 279, $182-186$

(20) Jiang, T.; Chang, Y. H.; Zhao, G. Y.; Han, B. X.; Yang, G. Y Synthetic Commun, 2004, 34, 225-230.

(21) Marcilla, R.; de Geus, M.; Mecerreyes, D.; Duxbury, C. J. Koning, C. E.; Heise, A. Eur. Polym. 2006, 42, 1215-1221.

(22) Lee, S. H.; Dang, D. T.; Ha, S. H.; Chang, W. J.; Koo, Y M. Biotechnol. Bioeng. 2008, 99, 1-8.

(23) Crowhurst, L.; Falcone, R.; Lancaster, N. L.; Llopis-

Mestre, V.; Welton, T. J. Org. Chem. 2006, 71, 8847-8853.

(24) Ranieri, G.; Hallett, J. P.; Welton, T. Ind. Eng. Chem. Res. 2008, 47, 638-644

(25) van Meter, D. S.; Yaqin, S.; Parker, K. M.; Stalcup, A. M.

Analytical and Bioanalytical Chemistry 2008, 390, 897-905.

(26) Kobrak, M. N. Green Chem. 2008, 10, 80-86.

(27) Abraham, M. H.; Acree, W. E. Green Chem. 2006, 8, 906

915 6th ed.; Prentice Hall, 1986.

(29) Trulove, P. C.; Osteryoung, R. A. Inorg. Chem. 1992, 31,

3980-3985.

$(30)$

(31) Crowhurst, L.; Mawdsley, P. R.; Perez-Arlandis, J. M.; Salter, P. A.; Welton, T. Phys. Chem. Chem. Phys. 2003, 5, 2790-2794. 